\title{
Study on the Layout of 15-Minute Community-Life Circle in Third-Tier Cities Based on POI: Baoding City of Hebei Province
}

\author{
Zeqin Li, Jie Zheng, Yukun Zhang \\ School of Architecture, Tianjin University, Tianjin, China \\ Email:820478679@qq.com, tjuzhengjie@163.com
}

How to cite this paper: Li, Z.Q., Zheng, J. and Zhang, Y.K. (2019) Study on the Layout of 15-Minute Community-Life Circle in Third-Tier Cities Based on POI: Baoding City of Hebei Province. Engineering, 11, 592-603.

https://doi.org/10.4236/eng.2019.119041

Received: August 20, 2019

Accepted: September 14, 2019

Published: September 17, 2019

Copyright (c) 2019 by author(s) and Scientific Research Publishing Inc. This work is licensed under the Creative Commons Attribution International License (CC BY 4.0).

http://creativecommons.org/licenses/by/4.0/

\begin{abstract}
At present, the planning of 15-minute community-life circle is being actively promoted in China. Taking Baoding City as an example, the status quo of service facilities layout was analyzed based on the POI data. Using the spatial accessibility algorithm, the ranges of 15-minute life circle of more than 1000 communities in Baoding were determined. By calculating the up-to-standard rates of public service facilities in each district, it is found that the allocation of medical facilities and commercial facilities in urban community life circle is relatively perfect, but the allocation of public cultural facilities and pension facilities is obviously inadequate. Based on this, optimization suggestions on the layout of public service facilities in the 15-minute life circle were put forward from three aspects: facilities sharing, function mixing and population structure and activity characteristics differentiation. It not only provides data and technical support for the planning and construction of community life circle in Baoding city, but also provides referable examples and promotable models for the community planning of third-tier cities.
\end{abstract}

\section{Keywords}

Community Life Circle, Accessibility, Up-to-Standard Rate, Planning Strategy

\section{Introduction}

In recent years, the concept of "15-minute life circle" has been widely recognized and promoted in China, and gradually applied to urban planning. In 2016, the Shanghai Master Plan put forward the idea of "15-minute community life circle" as the basic unit of building community life, providing basic service functions 
and public activity space for residents within walking distance, aiming to form a safe, friendly and comfortable community living platform [1]. The newly promulgated Standard for Urban Residential Area Planning and Design (GB 50180-2018) in 2018 clearly defines the meaning of " 15 -min pedestrian-scale neighborhood". According to the principle of the basic living needs of residents, facilities are allocated within the reasonable distance of 15-minute walking. In this context, it is imperative to implement the planning of urban "15-minute life circle". Because of the large number of third and fourth-tier cities and the large population of those cities, and the relatively outdated infrastructure, those cities are the focus of urban development planning. Taking Baoding City as an example, this paper explores the life circle planning which is still in its infancy.

The research and planning of life circle began in Japan in the 1960s. In 1969, the Ministry of Construction and the Ministry of Land Affairs of Japan put forward the concepts of "local life circle" and "settled circle". Settlement circle was dominated by people's demand for activities. According to residents' daily life needs such as employment, schooling, shopping, medical treatment, education and entertainment, area coverage for planning a day's life was defined as a spatial planning unit. Later, this concept was gradually accepted in Asia. South Korea's residential planning was affected by it, and the allocation of community service facilities was organized with the construction of different levels of life circles. In the comprehensive development plan of 1979, Taiwan also adopted the concept of "local life circle" to classify cities and promote balanced regional development [2].

In recent years, the concept of "circle of daily life" corresponding to community level has been gradually attached importance in China. It has been used in the research and planning practice of built environment assessment and public facilities allocation. Xiong Wei et al. [3] took Nanjing as an example to divide the life circle and select public facilities factor such as public service facilities and public space to evaluate the suitability of human settlement environment. Wu Qiuqing [4] discussed the allocation renewal strategy of community service facilities in mega-cities Megacities from the Living Circle Perspective. Zhao Yanyun et al. [5] compared the differences of public service facilities development in different urban areas of Beijing by measuring the configuration of public service facilities within the 15-minute living circle. Taking Beijing as an example, Chai Yanwei et al. [6] discussed the equalization strategy of public services in different strata and regions based on the spatial and temporal behavior of urban residents. Taking Guangzhou as an example, Xiao Jinghao et al. [7] evaluated the allocation of public facilities in community life circle based on the principle of decision tree. A large number of studies have confirmed that life circle, from the perspective of residents' living space, combined with urban and rural planning, has become an important tool to balance resource allocation, maintain spatial justice and organize local life. There are a lot of researches that focus on the firstand second-tier cities in China. However, the third and fourth-tier cities are also 
the main body of China's development and the "short slab" of modern city construction. Therefore, this paper focuses on the allocation of public service facilities in the third-tier urban life circle, and combines with relevant research to explore the optimal strategies for the allocation of public service facilities in the third-tier cities, taking Baoding City, Hebei Province as an example.

At present, there are two main methods to measure life circle: one is based on survey of residents' daily activities. For example, Sun Daosheng et al. [8] have spatially divided the community life circles by collecting GPS activity trajectories of individual residents in 18 communities. Although this method pays attention to the real behavior information of residents, it may be affected by the behavior habits of some residents, which leads to the deviation of the survey results. The second is to use the analysis method of the buffer. For example, Zhao Yanyun et al. [5] take the entrance of the community as the starting point and $1 \mathrm{~km}$ as the radius (15 minutes walking distance), the buffer zone is constructed as the scope of community life circle and the spatial distribution of public service facilities in the community is calculated. However, this method does not take into account the real road network situation, so that the results are not accurate enough. In this study, the existing methods are synthetically used for reference, and the GIS software platform is used to construct the traffic network data set to calculate the real walking path of residents. This method breaks through the limitation of buffer calculation with a certain distance, and also makes the results more authentic.

\section{Data Acquisition}

The data collected by the research institute mainly include the datasets of administrative regions, POI data of public service facilities, road data at all levels, and related data of residential districts in downtown Baoding. The data types and sources are shown in Table 1.

Among them, the POI of public service facilities (Point of Interest, refers to the point containing the information of the name, category, geographical location of facilities) is crawled on the Amap using Python tool, and the time node is June 2019. Referring to the Standards for Planning and Design of Urban Residential Areas (GB 50180-2018) and the daily needs of community residents, these data can be divided into six categories: convenient commercial facilities, educational facilities, medical facilities, recreational and sports facilities, dining facilities and pension facilities, shown in Table 2. After data acquisition, cleaning (eliminating invalid information such as errors and duplication) and coordinate

Table 1. Data resources.

\begin{tabular}{cccc}
\hline Data Content & Data Resource & Data Content & Data Resource \\
\hline Administrative region & Shuijingzhu Map & $\begin{array}{c}\text { POI data of public } \\
\text { service facilities }\end{array}$ & Amap \\
City road network & Shuijingzhu Map & Dataset of communities & Website of Anjuke lnc.
\end{tabular}


Table 2. Categories of facilities.

\begin{tabular}{|c|c|c|}
\hline Category & Specific Facilities & Quantity \\
\hline Convenient commercial facility & $\begin{array}{l}\text { Convenience store, super market, vegetable market, } \\
\text { greengrocery }\end{array}$ & 2898 \\
\hline Dining facility & $\begin{array}{l}\text { Chinese restaurant, fast-food restaurant, casual } \\
\text { restaurant }\end{array}$ & 11871 \\
\hline Educational facility & Kindergarten, primary school, high school & 438 \\
\hline Medical facility & Drugstore, clinic, general hospital & 1542 \\
\hline Recreational and sports facility & $\begin{array}{l}\text { Public recreational building, sports building, } \\
\text { community cultural center }\end{array}$ & 530 \\
\hline Pension facility & Nursing home, recreation center for the aged & 102 \\
\hline
\end{tabular}

rectification (because the original data coordinate system is Mars coordinate system, coordinate rectification needs to be converted to WGS84 coordinate system), it is imported into GIS for research, shown in Figure 1.

\section{Research Methods}

\section{1) Kernel Density Analysis}

Kernel density analysis is used to calculate the density of elements in their surrounding fields. It can be expressed as $f(x)=\frac{1}{n h^{d}} \sum_{i=1}^{n} K\left(\frac{x-x_{i}}{h}\right) . K(x)$ is the kernel function, $h$ is the path distance attenuation threshold, i.e. bandwidth, $n$ is the sample points in the bandwidth range, $d$ is the dimension of the data $(d=2$ when calculating two-dimensional data), $\left(x-x_{i}\right)$ represents the distance from the estimated point to the sample point $x_{i}$. In the kernel density analysis, the weight of the points in the search area is in reverse proportion to the distance from the grid search center, that is, the closer to the grid search center, the greater the weight of the points. The kernel density analysis tool in GIS is used to analyze the data of various facilities in urban area of Baoding, and classifies the output results by the natural breakpoint classification method.

\section{2) Reachability Analysis}

The data sets of road network in Lianchi and Jingxiu districts of Baoding are constructed using Arcgis software, and the service area analysis in network analysis tool is used to simulate all the reachable locations that residents living in 1113 communities in Baoding downtown area can walk to in 15 minutes. Therefore, 1113 15-min pedestrian-scale life circles are formed. 15-min life circle elements and POI data of each district were spatial joined, and the up-to-standard rate of facilities in each district was calculated. The flow chart is shown in Figure 2. The difference between this method and buffer analysis method is that it can reflect the travel status of residents to the greatest extent, shown in Figure 3.

\section{3) Up-to-Standard Rate of Facilities in Communities}

For any community, the " 15 -minute life circle" standard is directly related to 


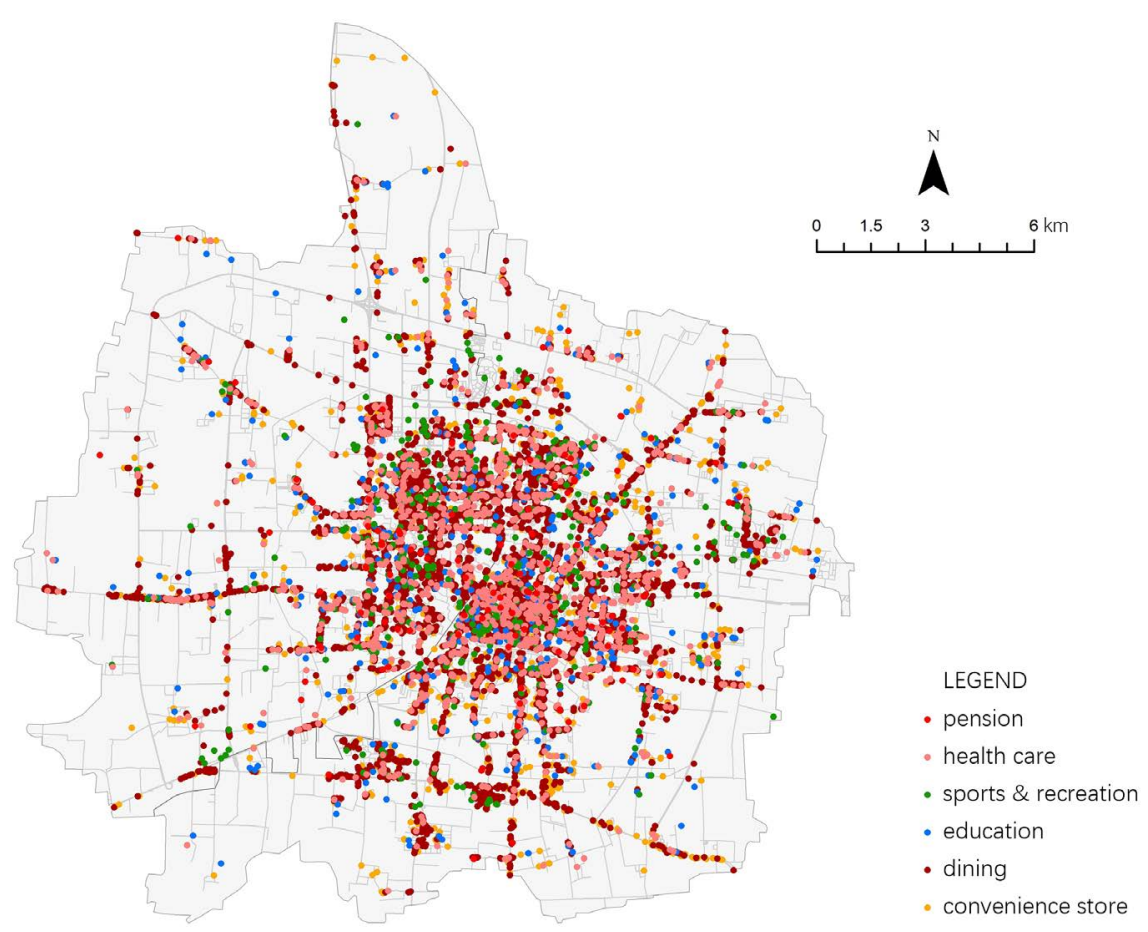

Figure 1. POI Dataset of facilities.

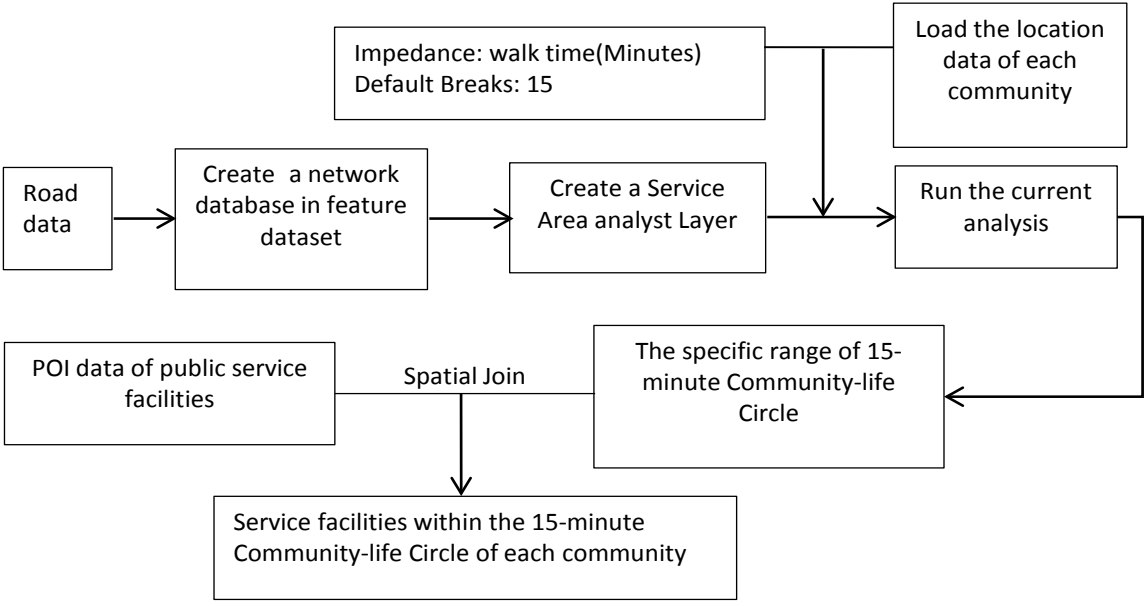

Figure 2. The flow chart of the spatial accessibility algorithm.

the quality of residents' daily life. Before calculating the up-to-standard rate of facilities, it is necessary to define the standard according to the needs of residents for different facilities. Its definition is as follows: 1) the overall standard: in categories of convenience business, dining, education, medical treatment, sports \& recreation and pension, at least one specific facility is needed to meet the standard. 2) Sub-category standard: considering the substitution between facilities (e.g. Chinese restaurant and fast-food restaurant have strong substitutability, while kindergartens, primary schools and high schools are educational facilities for different age groups. There is no substitutability between them, and they need to exist at the same time). 


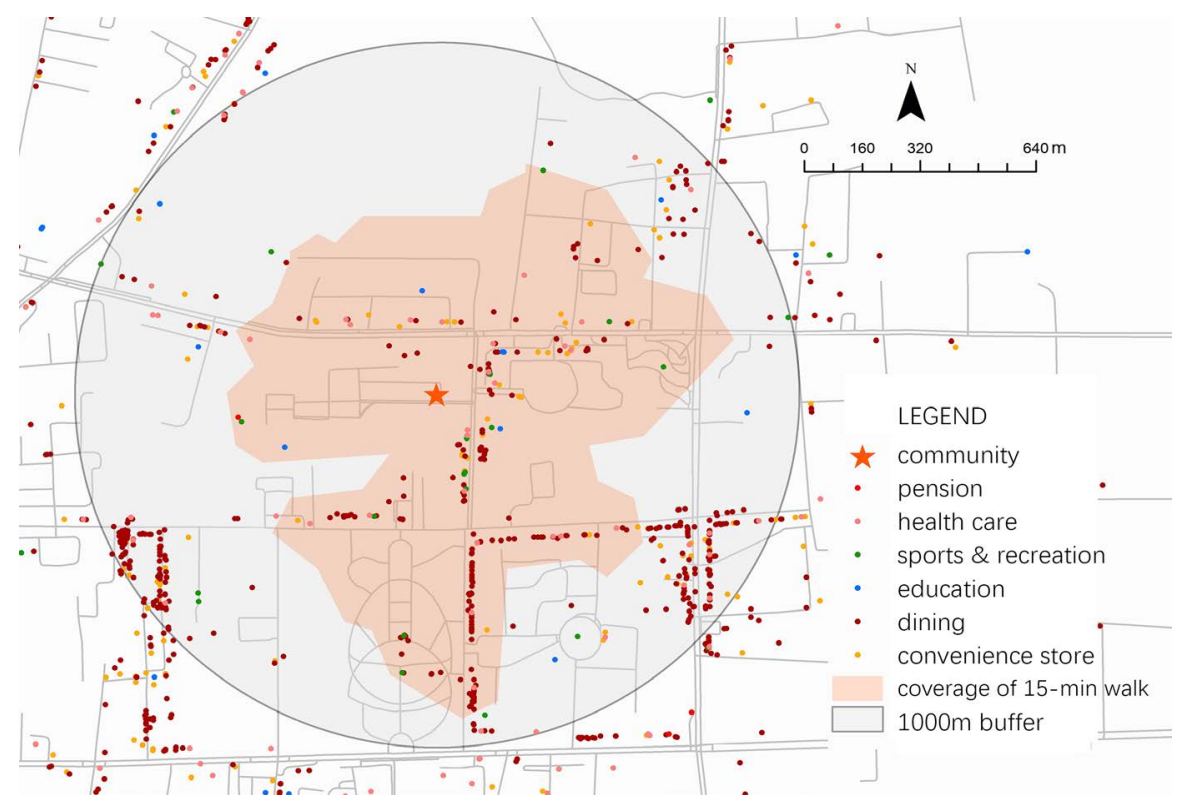

Figure 3. 15-min pedestrian-scale circle of a community.

\section{Results}

\section{1) Overall Layout of Facilities}

\section{a) Convenient Commercial Facilities}

As can be seen from the kernel density analysis map of convenience commercial facilities, the convenience commercial service facilities in Baoding are mainly concentrated in the downtown area. Places with the highest density are located in the Bell Tower, the Zhili Govern-general's Office and the East-West Street subdistrict, which were once the most prosperous areas in Baoding. In addition, there are many small-scale kernel districts, because apart from the traditional downtown commercial districts, major residential functional districts are forming their own commercial service kernel districts, shown in Figure 4.

\section{b) Dining Facilities}

As can be seen from the kernel density analysis map of dining facilities, the distribution of dining facilities in Baoding is multi-kernel. These kernels locate in the business circles near Dacige, Wanbo Plaza, and around Xiantianxia-Baobai Shopping Square. The common feature is that it is the central area of residents' activities and the densely populated area in each district, shown in Figure 5.

\section{c) Educational Facilities}

Educational facilities have a wide spatial distribution, covering most areas of the research area. This shows that the spatial distribution of educational facilities is relatively balanced. Areas with the most abundant facilities are located in $\mathrm{Yu}$ hua subdistrict, Zhonghua Road subdistrict and Hepingli subdistrict, where advantages of educational resources are obvious, shown in Figure 6.

\section{d) Sports and Recreational Facilities}

The distribution of sports and recreational facilities in the central urban area 


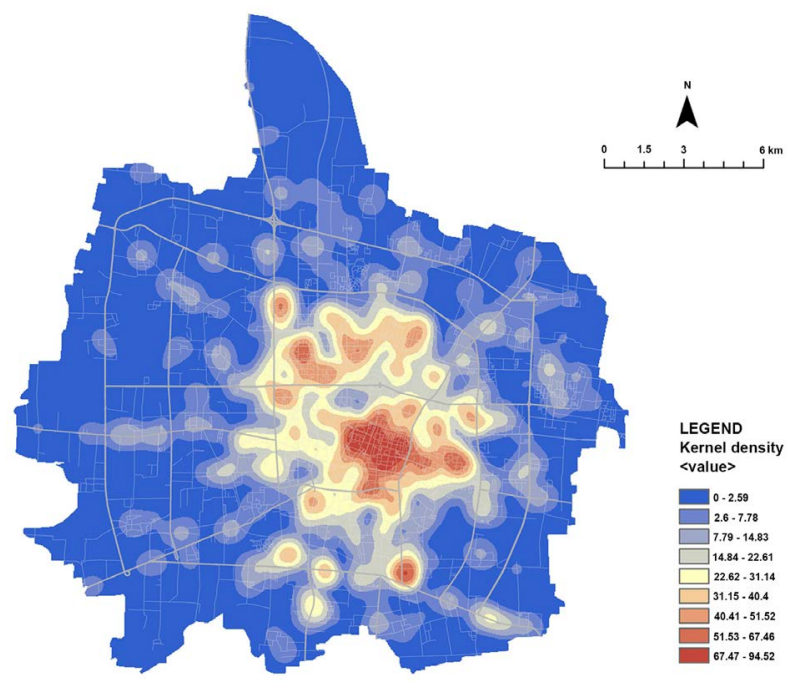

Figure 4. Kernel density of convenient commercial facility.

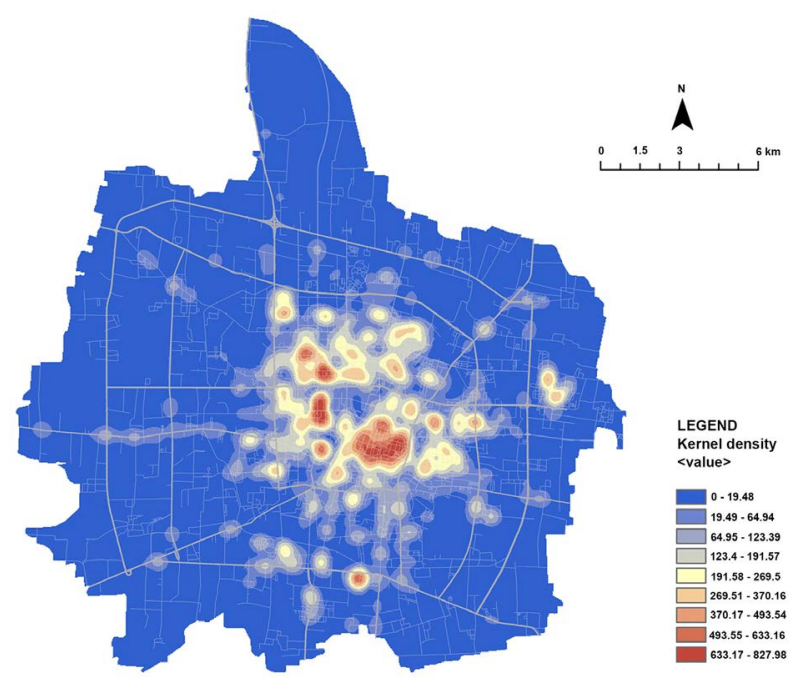

Figure 5. Kernel density of dining facility.

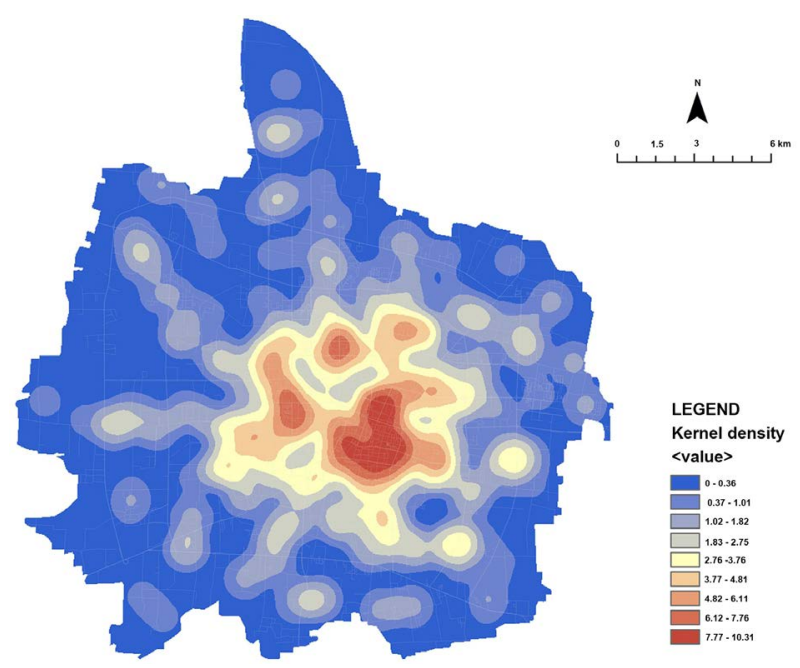

Figure 6. Kernel density of educational facility. 
is relatively uniform, and the regional differentiation is obvious. Higher density locations are concentrated in Yuhua subdistrict, Xianfeng subdistrict, North Hancun Road subdistrict and Hanzhuangxiang subdistrict. In terms of POI, the number of sports venues (409) is far more than that of public recreational venues (59), shown in Figure 7.

\section{e) Pension Facilities}

The number of POI of pension facilities (102) is relatively fewer, and there is a huge gap compared with other kinds of facilities. From its kernel density analysis, it can be seen that the core parts mainly locate in Hepingli subdistrict and Xinshichang subdistrict, and other places are scattered with some pension facilities, shown in Figure 8.

\section{f) Medical Facilities}

From the kernel density map of medical facilities, it can be seen that the density of urban center is high and shows a concentrated distribution, and regional deficiency can be seen around the Qiyi Road. In areas near the second and third ring road, medical facilities are only distributed along the main roads with a low density, shown in Figure 9.

\section{2) Up-to-Standard Rate of Facilities in Communities}

Six major categories are divided into 12 sub-categories, and calculated respectively, and in communities, the number of facility categories that meet the standard are calculated, shown in Figure 10 \& Figure 11.

As can be seen from Figure 10, the up-to-standard rate of convenient commercial facilities, dining facilities and medical facilities are very high, 98.65\%, $99.64 \%$ and $96.58 \%$ respectively. Although the total up-to-standard rates of educational facilities and sports facilities are high, the up-to-standard rates of sub-categories including primary schools (59.3\%), high schools $(43.31 \%)$ and public cultural facilities (50.22\%) are relatively low. The up-to-standard of total and sub-categories of pension facilities is poor.

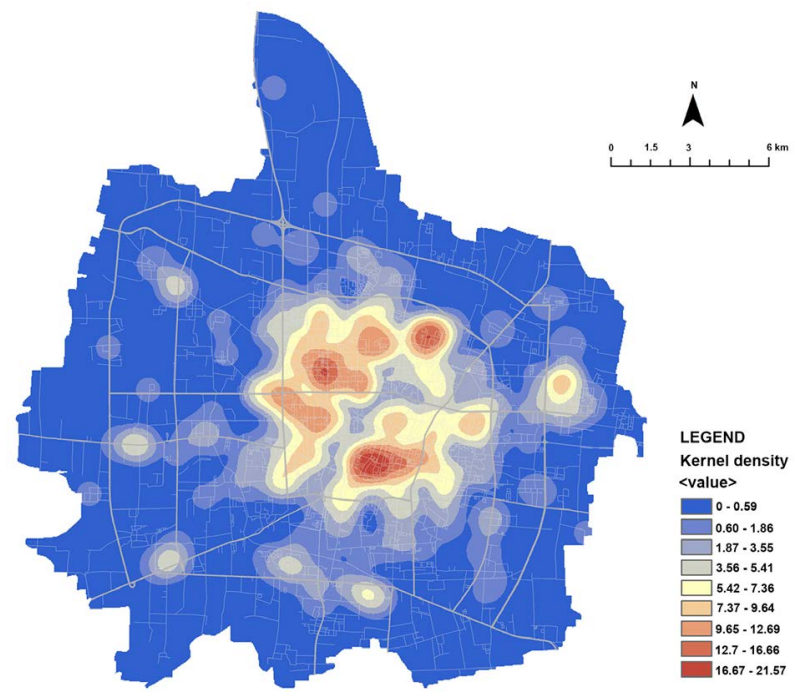

Figure 7. Kernel density of recreational and sports facility. 


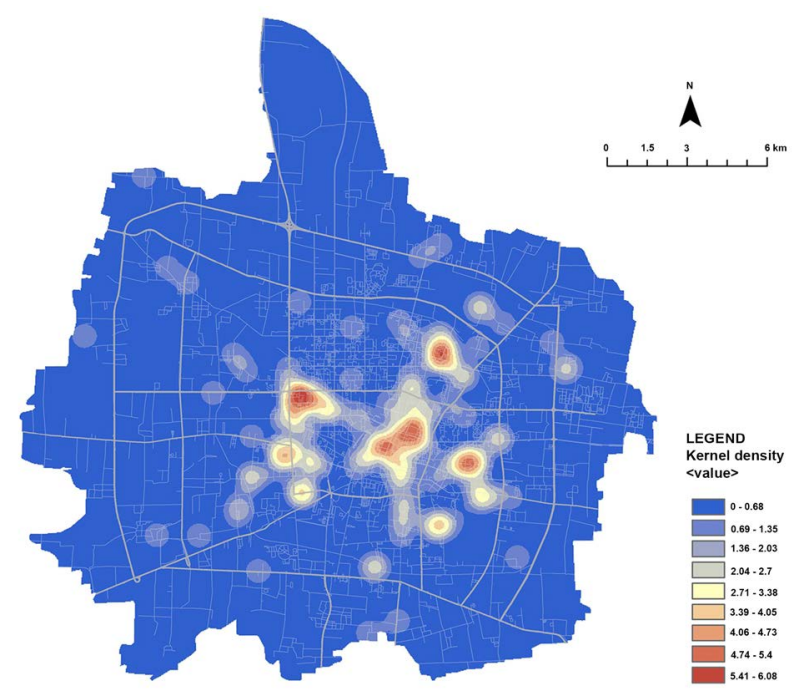

Figure 8. Kernel density of pension facility.

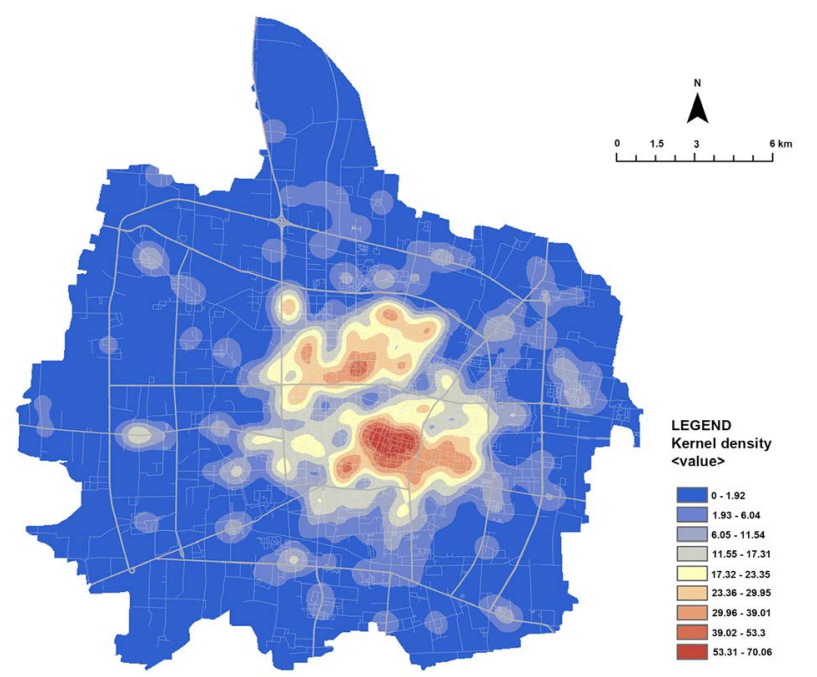

Figure 9. Kernel density of medical facility.

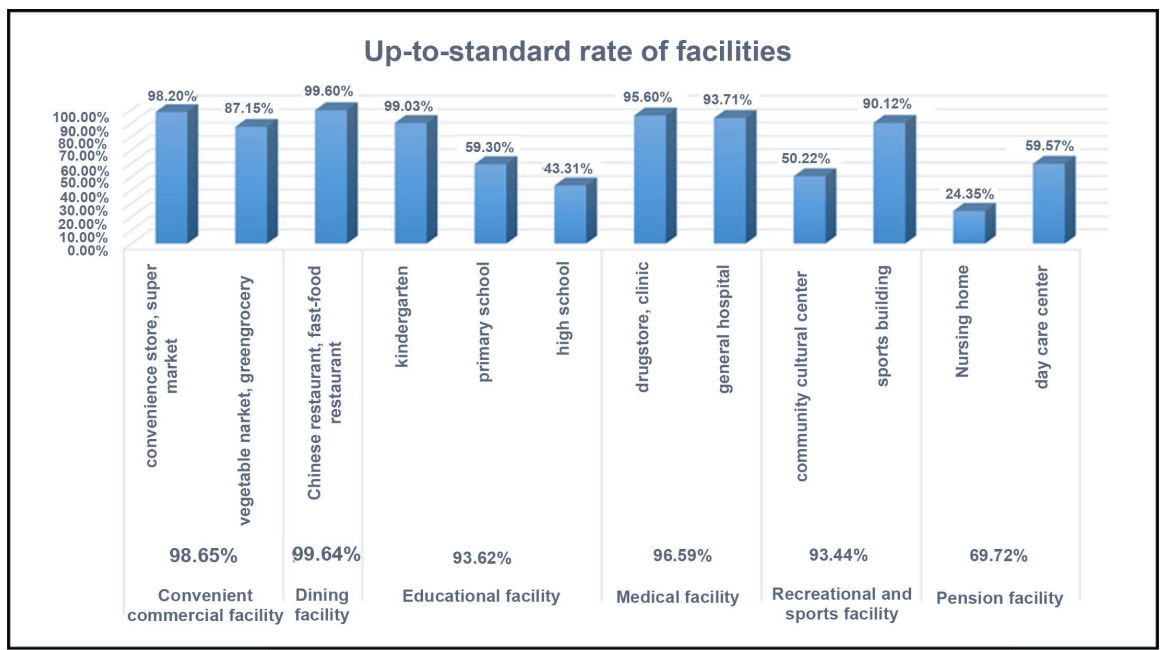

Figure 10. Up-to-standard rate of facilities. 


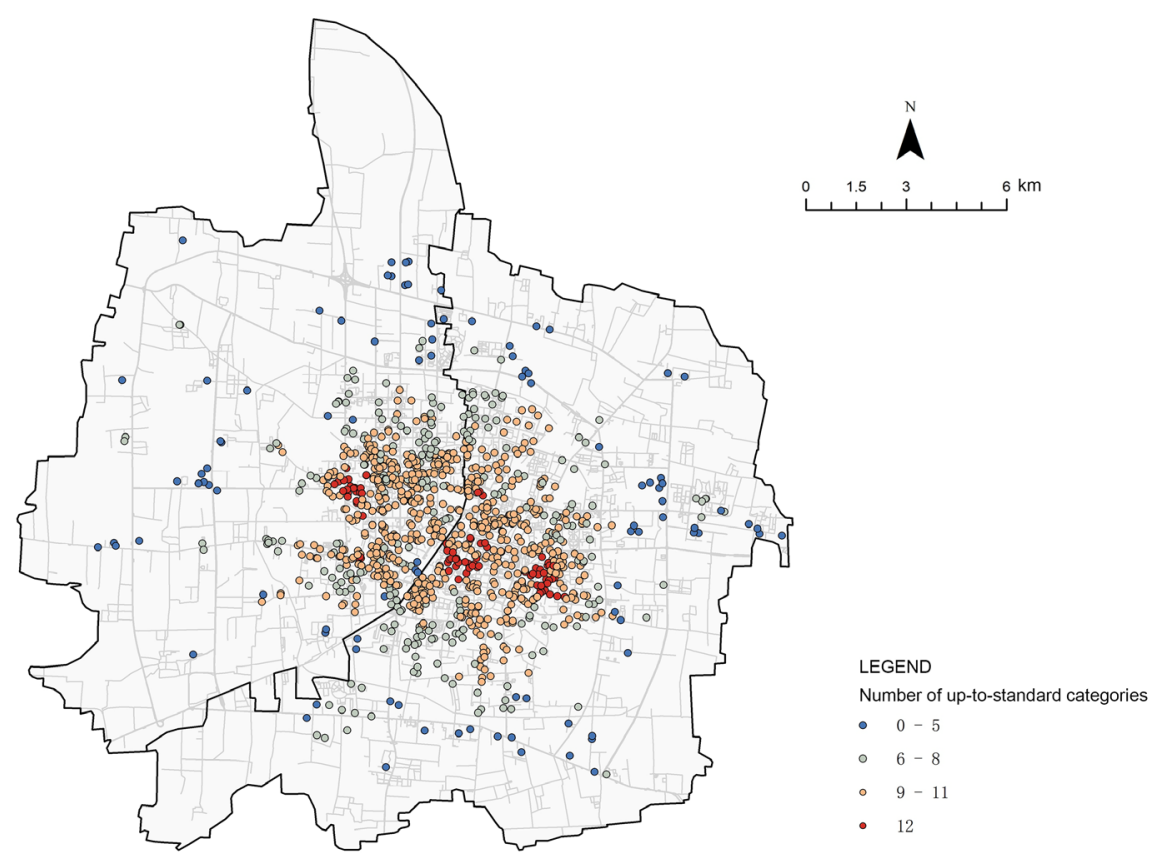

Figure 11. Number of up-to-standard categories.

According to the number of up-to-standard categories in communities, shown in Figure 11, only $7.63 \%$ of the 12 sub-categories have met the standard, and the average number of up-to-standard categories is 9. Among them, the nearer to the city center, the higher the up-to-standard rate is. The up-to-standard communities are mostly located in the vicinity of Qiyi West Road, Lekai North Street, Xiguan Street, Hengxiang South Street, Hongqi Street and Dongguan Street.

\section{Suggestions for Urban Planning}

\section{1) Facility Layout Strategy Based on Reachability and Share}

According to the up-to-standard rate of facilities, the overall allocation of community cultural facilities, pension facilities and some educational facilities in Baoding downtown area is insufficient. Therefore, it is necessary to improve the distribution of community cultural activities facilities, implement the indicators of pension services, and build educational facilities for all age groups. At the same time, according to the number of up-to-standard facilities in communities, we can accurately locate the missing facilities in each community, and then conduct precise construction.

For the more frequently used facilities, they shall be allocated in areas within 15 minutes of walk, therefore these facilities can be used by more communities and residents, and to a certain extent, form sharing to improve the service of facilities, shown in Figure 12.

\section{2) Layout Strategy with Mixed Functions}

Build a community graded living platform that matches the features of travel in the life circle, set up community neighborhood centers with different scales 


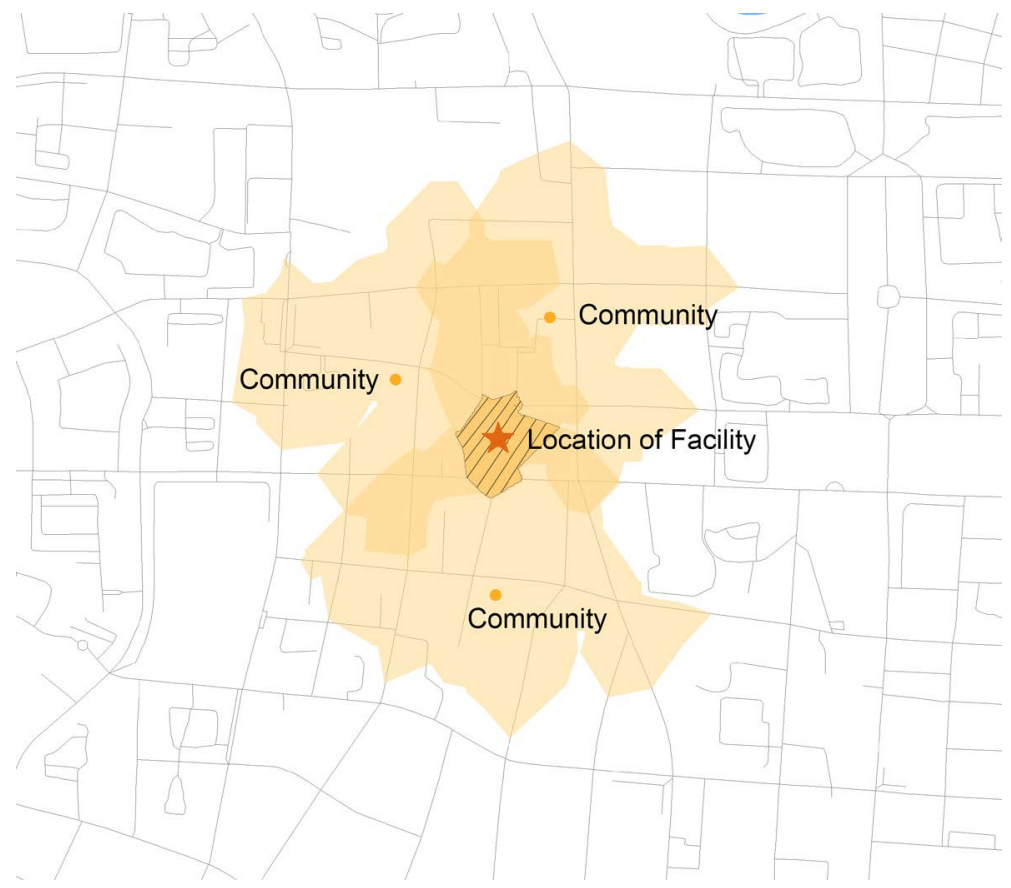

Figure 12. Facility distribution based on reachability and share.

and functions according to the size of the population, and combine the functions of community recreation, community pension, non-staple food market and other functions to form an intensive and complex layout form for community residents. Therefore, convenient and efficient "one-stop" life services are established to meet the different needs of residents, and high-quality and efficient community spaces are formed.

3) Differential Layout Strategy for Population Structure and Activity Features

Due to the differentiated, aging and highly educated people living in urban area, the lack of different facilities will bring different influences. While improving the facilities in the community life circle, we should also establish standards of public service facilities that suit the features of population structure. For example, the community with a higher population aging rate should be allocated with more pension service facilities. At the same time, the facilities should be rationally allocated according to the population density and residents' activities, the range of life circle can be expanded in the suburbs, where the population density is relatively low.

\section{Conclusion}

According to the research result of POI data of community public service facilities, in the 15-minute community life circle of Baoding downtown area, the allocation of convenient commercial facilities, dining facilities and medical facilities is relatively good; the allocation of community cultural facilities, primary and high schools needs to be improved; and the community pension service facilities need to be constructed urgently. In combination with the actual situation, 
the optimization suggestions for the layout of facilities are put forward. The third and fourth-tier cities are the main body of China's development and the "short slab" of modern city construction. Urban planners should seize the opportunity, pay attention to people's livelihood, quantitatively analyze the different needs of residents, implement the life circle, and build a safe, civilized, convenient and happy harmonious city.

\section{Foundation Project}

China's National Natural Science Foundation Project "Research on Green Productive Renewal Strategy and Method of Existing Urban Residential Areas" (51708395)

\section{Conflicts of Interest}

The authors declare no conflicts of interest regarding the publication of this paper.

\section{References}

[1] Shanghai Planning and Land Resources Administration (2016) Shanghai Planning Guidance of 15-Minute Community-Life Circle. http://ghzyj.sh.gov.cn/zcfg/ghss/201609/t20160902_693401.html

[2] Xiao, Z.P., Chai, Y.W. and Zhang, Y. (2014) Overseas Life Circle Planning and Practice. Planners, 10, 93-94.

[3] Xiong, W. and Xu, Y.L. (2010) The Urban Settlement Research in the Perspective of Public Facilities: A Case Study of Nanjing. Modern Urban Research, 12, 35-42.

[4] Wu, Q.Q. (2015) The Exploration on the Dynamic Programming of Community in Megacities from the Living Circle Perspective. Shanghai Urban Planning Review, 4 13-19.

[5] Zhao, Y.Y., Zhang, B. and Zhou, F. (2018) Study on the Spatial Measurement of "15-Minute Community Living Circle" in Beijing Based on POI. The World of Survey and Research, 5, 17-24.

[6] Chai, Y.W., Zhang, X. and Sun, D.S. (2015) A Study on Life Circle Planning Based on Space Time Behavioural Analysis: A Case Study of Beijing. Urban Planning Forum, 3, 61-69.

[7] Xiao, J.H., Zhou, D.L. and Hu, J.P. (2018) Measurement And Evaluation Method of Community Life-Cycle Based on Decision Tree Theory: Panyu District of Guangzhou. Planners, 3, 91-96.

[8] Sun, D.S., Chai, Y.W. and Zhang, Y. (2019) The Definition and Measurement of Community Life Circle: A Case Study of Qinghe Area in Beijing. Urban Studies, 23, 1-9. 